\title{
Estudio exploratorio de mercado para Lippia alba como alternativa de producción sostenible en la cuenca media y baja del Rio Las Ceibas, Neiva Colombia
}

\section{Exploratory market study for Lippia alba as an altermative sustainable production in the middle and lower basins of the Las Ceibas River, Neiva Colombia}

\author{
Sandra Vianney Fajardo ${ }^{[1]}$ y Pavel Tovar Lizcano ${ }^{[2]}$
}

\begin{abstract}
Resumen
Las plantas medicinales son un componente importante del recurso forestal no maderable del bosque, que puede contribuir al desarrollo sostenible de los habitantes presentes en las áreas protegidas. Según diversos autores el mercado mundial de las plantas medicinales se valora en US 47 millones; sin embargo, en Colombia su comercialización prevalece la informalidad, lo que dificulta estimar su valor e impacto en las comunidades rurales.

En la cuenca del río Las Ceibas que abastece de agua la ciudad de Neiva, se encontró que la planta Lippia alba (Mill.) N.E.Br es un recurso forestal no maderable que con fines medicinales posee el mayor potencial para el aprovechamiento sostenible de los habitantes de este ecosistema estratégico. Por lo anterior resulto oportuno realizar un estudio exploratorio de mercado y elaborar una propuesta de producción sostenible de Lippia alba como alternativa estratégica para la gestión de la cuenca media y baja del río Las Ceibas. Se identificaron las limitaciones y oportunidades que tienen los habitantes de la cuenca media y baja del río Las Ceibas para incursionar con Lippia alba en la cadena productiva de las plantas medicinales en Colombia, así como también, los diferentes actores institucionales que pueden contribuir en este proceso y las posibilidades que a partir de un desarrollo agroindustrial a mediano y largo plazo se pueda incursionar en el mercado nacional e internacional de sus aceites esenciales.
\end{abstract}

Palabras clave: Mercado de plantas medicinales; Lippia alba; Agroindustria; Cuenca del río Las Ceibas.

\begin{abstract}
Medicinal plants constitute an important component of the non-timber forest resource that could contribute to the sustainable development of the inhabitants of the protected areas. According to various authors, the global market for medicinal plants is valued at approximately US\$ 47 million; however, in Colombia the
\end{abstract}

[1] M.Sc. Ecología y Gestión de Ecosistemas Estratégico. SE Neiva, Universidad Surcolombiana. fajardosandra@gmail.com [2] M.Sc. Ecología y Gestión de Ecosistemas Estratégico. Universidad Surcolombiana, SE Huila. paveltl@gmail.com

Recibido: 30 abril 2015 • Aceptado: 15 mayo 2015. 
commercialisation of such plants is largely informal, making it difficult to estimate their value and impact on the rural communities.

In the Las Ceibas River basin, that supplies water to the city of Neiva, we found the Lippia alba (Mill.) N.E.Br medicinal plant to present the greatest potential for sustainable exploitation for the inhabitants of this strategic ecosystem. Given the above, we undertook an exploratory market study and elaborated a proposal for the sustainable production of Lippia alba as a strategic alternative for the management of the middle and lower basins of the Las Ceibas river. We identified the limitations and opportunities for the inhabitants of the middle and lower basins of the Las Ceibas river to introduce Lippia alba in the productive chain of medicinal plants in Colombia. We also identified the different institutional actors that could contribute to this process and the possibilities of introducing the plant's essential oil into the national and international market, based on medium- and long-term agroindustrial development.

Key words: medicinal plan market; Lippia alba; Agroindustry; Las Ceibas River basin.

\section{Introducción}

La importancia de la cuenca del río las Ceibas, está en su función como abastecedora del acueducto del municipio de Neiva, de manera que el grupo de investigación Ecosistemas Surcolombianos (ECOSURC) de la Universidad Surcolombiana ha reconocido su importancia como ecosistema estratégico, en términos de la existencia de asentamientos humanos; la producción de agua para el consumo humano; defensas y amenazas naturales; la posibilidad de realizar actividades educativas y científicas; su diversidad natural; el reconocimiento legal y académico; y la producción de agua y suelo para la agricultura (Patiño, 2004; Olaya, 2003; Olaya y Sánchez, 2003).

Frente a la anterior situación, la Corporación Autónoma Regional del Alto Magdalena CAM (2006) señala que es necesario fomentar el desarrollo sostenible de los recursos naturales presentes en la cuenca, en consecuencia, propone que el recurso forestal no maderable puede constituirse en la base para establecer un sistema de aprovechamiento sostenible en la cuenca del río Las Ceibas. En este orden de ideas, un estudio realizado por Fajardo (2014) encontró que la planta Lippia alba (Mill.) N.E.Br. posee el mayor potencial para el aprovechamiento sostenible de un recurso forestal no maderable con fines medicinales en la cuenca media y baja del río Las Ceibas.

Entre los recursos forestales no maderables la riqueza de las plantas medicinales, asociada al conocimiento ancestral de su uso etnofarmacológico, se presentan como una alternativa natural y de bajo costo para el tratamiento y prevención de enfermedades, frente al alto costo de medicamentos manufacturados; de manera que el mercado mundial de las plantas medicinales tiene un valor estimado en US 47 millones anuales. Sin embargo, la comercialización de estos productos mantiene un halo de informalidad que dificulta su valoración económica e impacto sobre las comunidades rurales (Puelles, 2008; R. Martínez, 2006; G. Martínez, 2005; Galy et al, 2000).

Colombia podría ser más competitivo en el aprovechamiento de las plantas medicinales y de los productos derivados de estas. Lo anterior señala parte del futuro del programa de mercados verdes, que se caracteriza por tranzar productos que se destacan por sus consideraciones ambientales, generando un valor agregado que reconocen los consumidores (Guerrero, 2000; Van Hoof, 2000). En este sentido las plantas medicinales se constituyen en alternativa sostenible de mercado, en la cual, la agroindustria produce materia prima como es el caso de los aceites esenciales, colorantes y fitofármacos. De aquí surge la importancia social y económica, que mediante investigaciones de los principios bioactivos, en agroindustria y de mercado puedan impulsar la economía de una región (Vega, 2001).

Cabe agregar, que las investigaciones realizadas en torno a las plantas medicinales, se han centrado en aspectos etnobotánicos y en la identificación de componentes bioactivos y pocos trabajos abordan aspectos como cadenas de valor, la distribución de beneficios e impacto del comercio en los ecosistemas y en comunidades rurales, así como tampoco, en la capacidad productora y de comercialización (R. Martínez, 2006). Además, para desarrollar la comercialización y el establecimiento de mercados, es necesario establecer procesos agroindustriales que 
lleven a mejorar la calidad en la etapa de producción y transformación (Palacios, 2014).

Por las consideraciones anteriores y teniendo como base lo obtenido por Fajardo (2014), resulto oportuno realizar una investigación que identificara las posibilidades que tiene el recurso Lippia alba. para ingresar a la cadena de las plantas medicinales en Colombia y proponer una estrategia que contribuya a la consolidación del plan de gestión de la cuenca río Las Ceibas mediante el aprovechamiento sostenible de este recurso forestal no maderable del bosque.

\section{Métodos}

Para identificar las posibilidades que tiene Lippia alba para ingresar a la cadena de las plantas medicinales en Colombia se realizó un estudio exploratorio de mercado. Para esto, se desarrolló un trabajo de campo que consistió en una entrevista en profundidad, dirigida a 21 comerciantes presentes de los principales mercados de plantas medicinales en la ciudad de Neiva. Estas entrevistas al ser un mecanismo flexible, abierto, no directo y dinámico, es una conversación entre iguales, y no un intercambio de preguntas y respuestas formales (Cardona, 2005), permitieron la comprensión de la dinámica comercial de las plantas medicinales y de Lippia alba en el ámbito local, a partir de la experiencia comercial de los entrevistados.

De manera paralela se llevó a cabo un análisis de información secundaria de los documentos de Barrientos et al (2012), DANE (2011), el Consejo Nacional de Política Económica y Social y el Departamento de Planeación Nacional de la República de Colombia (2010), López et al. (2009), Mesa-Arango et al (2009), Gómez (2008), LATINPHARMA (2008), Nope et al (2008), DANE (2007), Durán et al (2007), Ciccio et al (2006), Díaz (2006) ICONTEC (2005), (UNITED NATIONS CONFERENCE ON TRADE AND DEVELOPMENT, 2005), Gutiérrez et al (2004), BIOCOMERCIO SOSTENIBLE (2003), Díaz (2003), PROEXPORT (2003), Ocampo y Valverde (2000).

A partir de lo anterior se realizó un análisis de oferta y demanda a nivel nacional e internacional; se estableció el comportamiento de costos y análisis competitivo, se identificaron las posibilidades de sostenibilidad económica, se definieron los obstáculos, limitaciones y condiciones normativas que se deben superar; se describieron las características del mercado de Lippia alba y se evaluaron sus posibilidades de ingreso a la Cade- na Productiva de las plantas Aromáticas, Medicinales, Condimentarías y Afines en Colombia (PAMC) con base en los conceptos desarrollados por García y Polanía (2007) Benassini (2001), Kinnear y Taylor (1998), Ferré y Ferré (1997).

Para la propuesta de gestión se emplearon los resultados generados en los pasos anteriores, a partir del análisis de los componentes ecológico, social e institucional como lo concibe López et al (2009), Baker (2008) y los principios del enfoque por ecosistemas de la CDB (2007; 2004). Por esta razón se plantean ejes operacionales relacionados con la estructura social, el modelo de producción sostenible, la transferencia tecnológica junto con la transformación y comercialización del producto.

\section{Resultados y discusión}

\section{Marco reglamentario del mercado de las plantas medicinales}

Para la incorporación de Lippia alba a la cadena productiva de las plantas medicinales, se debe tener en cuenta y aplicar dentro de la producción y comercialización la legislación nacional, que cuenta con principios y normas orientadas a la conservación, conocimiento, uso sostenible y mejoramiento de la biodiversidad y protección del medio ambiente, para defender la salud y el bienestar de los Colombianos amparados en la Ley 23 (1973) y el Decreto 2811 (1974) por los cuales se expide y reglamenta el Código de Recursos Naturales y protección al medio ambiente y la Ley 165 (1994) por medio de la cual se aprueba el Convenio sobre la Diversidad Biológica.

Además, el estado Colombiano ha establecido normas que tienen en cuenta la provisión de salud y al mismo tiempo el cuidado del ambiente, como son la Ley 86 (1993) que estipuló la regulación del uso e industrialización de la flora medicinal y la Resolución 05078 (1992) que adopto normas en materia de medicinas tradicionales y terapéuticas. Por otra parte el Ministerio de Salud mediante el Decreto 677 (1995) estableció el registro INVIMA para controlar los laboratorios de medicamentos, cosméticos, preparaciones farmacéuticas a base de recursos naturales (fitoterapéuticos).

Las Buenas Prácticas de Manufactura según el Decreto 3075 (1997) del Ministerio de Salud, establece normas, procesos y cimientos técnicos para garantizar la calidad de los productos en el proceso de 
comercialización de recursos naturales elaborados por los laboratorios naturistas y fitofarmacéuticos. Del mismo modo, se cuenta con la norma técnica Colombiana NTC 5400 de buenas prácticas agrícolas para frutas, hierbas aromáticas, culinarias y hortalizas frescas (BPA), que busca garantizar la inocuidad de los productos agrícolas en Colombia y lograr la sostenibilidad ambiental, económica y social (ICONTEC, 2005).

\section{Análisis del mercado para Lippia alba (Pronto alivio)}

La importancia económica y valor comercial de Lippia alba radica en su comercialización en plazas de mercado en fresco o seco, y en menor proporción en laboratorios fitofarmacéuticos y cosméticos para extraer sus aceites esenciales (Cicció et al., 2006; Díaz 2006, 2003; PROEXPORT, 2003).

\section{Demanda}

Dentro de las variedades de Lippia alba que se presentan a nivel mundial se encontró que son plantas de una misma especie que se diferencian entre sí porque presentan una distinta composición química del aceite esencial, de los cuales se conocen que tienen mayor movimiento en el sistema de información de comercio exterior al quimiotipo citral, el quimiotipo carvona y el quimiotipo híbrido que contiene carvona y limoneno (Cicció y Ocampo, 2006; Gómez, 2008; Durán et al 2007; Mesa-Arango et al, 2009). Según la Asociación Latinoamericana de Integración (ALADI) los mayores productores de citral son Alemania, EEUU, Brasil, España y China y los productores de limoneno son Brasil, EEUU, Alemania, España, Reino Unido, Francia y Suiza. De acuerdo con el mismo organismo, Colombia reporta importaciones de citral desde el año 2007 de países como Alemania, Brasil, China, España, Estados Unidos, Francia, Indonesia, Italia, Japón, México, Países Bajos, Reino Unido y Suiza; y no reporta importaciones ni exportaciones de limoneno ni carvona, aceites esenciales que tienen gran movimiento a nivel internacional donde Brasil y México son los principales productores.

En el sector farmacéutico cerca del 42\% de los 25 medicamentos más vendidos en el mundo son de origen natural, mercado que tiene un valor estimado de más de 45 millones de Euros y continúa con un crecimiento firme. En este orden de ideas, el valor del comercio global de plantas medicinales es aproximadamente 10 millones de Euros anuales y tiene un crecimiento de un $10 \%$ anual; de estos mercados los más grandes se encuentran en Alemania, Francia, Italia, el Reino Unido y España (UNITED NATIONS CONFERENCE ON TRADE AND DEVELOPMENT, 2005). Según Gutiérrez et al. (2004) el $23 \%$ de los laboratorios de productos naturales en Colombia afirman que elaboran productos a base de Lippia alba, que en conjunto consumían 230 kilogramos mensuales. De la misma manera, se estableció que los precios de compra al proveedor eran de \$ USD 3.00 el kilogramo seco y de \$ USD 0.35 en fresco, con el fin de utilizar sus aceites esenciales como materia prima para elaborar especialmente te digestivo, comprimidos y encapsulados, sales para baños y cremas, entre otros. Dentro de este estudio se destaca el precio del aceite esencial de Lippia alba, que los laboratorios venden a razón de \$ USD 5.30 el frasco de $8 \mathrm{ml}$.

\section{Oferta}

En Colombia el mercado de las plantas medicinales está poco desarrollado y su mayor movimiento se da a través de mercados informales en los cuales no se registran sus valores transaccionales ni se genera información estadística al respecto. También se presenta que la producción de plantas medicinales no está vinculada a actividades de planeación de cultivos, y en consecuencia no hay certeza en la demanda mes a mes puesto que las plantas medicinales se comercializan en plazas de mercado, entre las cuales la Plaza Samper Mendoza en Bogotá mueve alrededor de 800 toneladas mensuales de plantas de todos los municipios de la región andina (Díaz, 2006). Esta informalidad comercial se encuentra asociada a la inexistencia de una organización de productores de plantas medicinales, y puede ser la causa del no cumplimiento de los estándares de calidad exigidos por en los laboratorios de productos naturales y mercados internacionales. La mayoría de los productores de plantas son campesinos que cultivan o recogen las plantas como una alternativa de diversificación a otros cultivos tradicionales. De la misma forma, no existen centros de acopio certificados por entidades regidas por el Ministerio de la protección social.

\section{Cadena productiva de Lippia alba}

En Colombia existen dos canales de mercadeo para Lippia alba, uno consiste en un sistema de comercialización en fresco para consumo directo que se realiza en las plazas de mercado de Medellín, Cali, Ibagué, Armenia, Manizales, Pereira, Villavicencio y 
Tocaima y los principales centros de acopio y comercialización en Bogotá y otro mercado en fresco o deshidratado para consumo por parte de los laboratorios de productos naturales (Díaz, 2006). En este sentido se encontró Lippia alba no tiene demanda en los mercados de la ciudad de Neiva, al ser una planta muy frecuente en las fincas cercanas y en los patios de los hogares de la ciudad.

La comercialización en fresco o deshidratado se realiza a través de 90 laboratorios. Éstos realizan procesos industriales para extraer los aceites esenciales de Lippia alba que son comercializados a través de diferentes marcas de productos fito-farmacéuticos, cosméticos y perfumes, que tienen como destino centros médicos, estéticos, centros naturistas y ventas por catálogo (Díaz, 2006; BIOCOMERCIO SOSTENIBLE, 2003). Según un informe presentado por la revista Dinero, en el año (2005) el mercado de las plantas medicinales en Colombia fue estimado en US\$23 millones, cifra que se considera representativa para un mercado incipiente que se encuentra en proceso de desarrollo y con tasas de crecimiento que oscilan alrededor del 10\%. Sin embargo, la sostenibilidad de este mercado es incierta puesto que sólo el 39\% de los productos se realizan con plantas que son cultivadas, un $10 \%$ proviene de productos importados y el restante $51 \%$ proviene de la extracción silvestre o de procesos de recolección del recurso natural (Díaz, 2006). Esto indica, que la mitad del mercado colombiano de los productos naturales, se provee de recursos naturales que son extraídos de los ecosistemas de los cuales hacen parte, situación que atenta con la conservación de los recursos naturales y llena de incertidumbre el futuro de dicho mercado, puesto que existe la posibilidad de no contar con materia prima para sus productos comerciales.

De manera adicional, y según Díaz (2006), este sistema de oferta de materia prima basado en la extracción silvestre y en la recolección del recurso, ha generado otra seria dificultad a los laboratorios de productos naturales, que consiste en la alta variabilidad de la oferta y la consecuente informalidad de las relaciones comerciales. Como puede observarse, esta situación revela la necesidad de establecer sistemas agroindustriales sostenibles que beneficien a los actores de la cadena productiva de la PAMC en Colombia. De acuerdo con las proyecciones de mercado propuestas por López et al. (2009), Gutiérrez (2004) y el Ministerio de Agricultura y Desarrollo Rural, y Biocomercio Sostenible (2003) los laboratorios de productos naturales para el año 2010 presentaron una demanda de Lippia alba deshidratada estimada en una 1.6 toneladas mensuales, lo que equivale a un consumo cercano a las 19 toneladas anuales deshidratadas de la planta. Este mercado según las proyecciones de Díaz (2006), Gutiérrez (2004) y Índice de Precios al Consumidor (IPC) del DANE (2011) tuvo un valor estimado de US\$79.600 en el año 2010.

No obstante, estas cifras no muestran la realidad del mercado nacional de Lippia alba, debido a que sólo hacen referencia a una pequeña fracción que corresponde al comercio formal realizado por los laboratorios y no muestran la comercialización que se realiza en las plazas de mercado que manejan volúmenes mensuales muy significativos (Díaz, 2006). Según los resultados obtenidos en el presente estudio, el ingreso de la producción de Lippia alba proveniente de la cuenca media y baja del río Las Ceibas puede darse mediante tres canales de distribución y comercialización. En el mercado interno se tienen dos posibilidades, una en las plazas de mercado y otra con los laboratorios de productos naturales. La tercera posibilidad de comercialización es el mercado internacional que demanda los aceites esenciales de la planta, el ingreso a este mercado es posible a largo plazo, en el cual se tendrán técnicas de cultivos apropiadas que garanticen la inocuidad del producto, óptimas concentraciones de aceites esenciales y la infraestructura instalada que permita la extracción de los mismos.

\section{Oportunidades y limitaciones de la cadena productiva del mercado de las PAMC}

El análisis del comportamiento de la cadena productiva de Lippia alba permitió identificar sus limitaciones y oportunidades. Según López et al. (2009) estas oportunidades y limitaciones se encuentran divididas por eslabones que se describen a continuación:

El mercado interno y externo presenta oportunidades como son que las plantas medicinales proveen opciones económicas atractivas para las comunidades ofreciendo posibilidades de desarrollo; que es un sector en pleno crecimiento en los últimos años, creando en especial oportunidades para el agronegocio de Lippia alba en la industria cosmética y farmacéutica; y que los ingredientes naturales de origen nativo tienen la oportunidad de colocarse en mercados de países como Estados Unidos, Alemania y Francia. Entre las limitaciones se tiene que el mercado no cuenta con información precisa sobre los cultivos y sus extractos, la demanda, oferta, ganancias y otros movimientos comerciales; que una limitante para el comercio for- 
mal es el cumplimiento que exige estándares de calidad del producto; por otro lado, en el sistema de información arancelaria no existen subpartidas específicas para muchos de los productos de plantas medicinales lo que dificulta conocer el movimiento en el comercio exterior.

Para los proveedores la condición nativa de Lippia alba en la cuenca media y baja del río Las Ceibas, se convierte en la principal oportunidad, puesto que ésta característica facilita la propagación, producción de la planta que permitiría alcanzar los estándares de calidad requeridos por la industria. Pero la falta de integración de los sistemas de producción, comercializadores y exportadores, les limita conocer las necesidades de los insumos requeridos de acuerdo con las proyecciones del mercado.

Los pequeños productores presentes en la cuenca media y baja del río Las Ceibas tienen la oportunidad de asociarse para abastecer la oferta de volúmenes y desarrollar procesos de transformación primaria de alta calidad y a partir de lo anterior de tener apoyo financiero, técnico e investigativo de los diversos actores presentes en la cadena productiva de Lippia alba. Como limitaciones tienen la prevalencia de prácticas inadecuadas en los cultivos, la falta de asistencia técnica, el desconocimiento de la normatividad y ausencia de manejo de cosecha y post-cosecha, aspectos que en conjunto dificultan ofrecer productos con los estándares de calidad exigidos por el mercado.

Para los transformadores las oportunidades radican en las tendencias de los consumidores, hacia el uso de productos a base de materia prima de extractos naturales sanos y diferenciados; pero esta misma tendencia les genera la principal limitación que consiste en la variable disponibilidad de materia prima, especialmente material vegetal crudo, por falta de inocuidad y constancia en las producciones.

En el ámbito institucional las oportunidades se basan en los diferentes escenarios de ingreso del sistema productivo de Lippia alba a la cadena productiva de plantas medicinales y aromáticas, además de las posibles alianzas de productores y transformadores que están establecidas dentro de la misma. Sin embargo, la falta de disposición política para llevar a cabo la disponibilidad presupuestal dirigida a proyectos rurales con enfoques de sostenibilidad y la poca o nula asistencia técnica prestada a pequeños y medianos productores es la dificultad mayor que se presenta desde lo institucional.
Sistema de producción sostenible de Lippia alba como alternativa estratégica para la gestión de la cuenca media y baja del río las ceibas

En cuanto a las condiciones agroecológicas, el cultivo de Lippia alba ha sido objeto de poca investigación, excepto algunas iniciativas desarrolladas en Costa Rica a nivel comercial. Según Ocampo y Valverde (2000) la planta soporta temporadas secas de hasta 4 o 6 meses y debe establecerse en sitios con buena radiación lumínica y con buenas condiciones de humedad lo cual hace que produzca mayor cantidad de ramas con hojas; por estas razones se recomienda sembrarla hasta los 1100 metros de altura, si se siembra a mayor altitud y en zonas frías, la planta crece con mayor dificultad. El mismo autor argumenta que esta especie es poco exigente en lo que respecta al suelo, sin embargo, para un buen establecimiento se requiere suelo bien drenado y media sombra. También recomienda hacer la colecta de la planta cuando el cultivo se encuentre en estado de floración y realizar su secado a la sombra, y así obtener mayores concentraciones de sus aceites esenciales.

Las anteriores condiciones agroecológicas se encuentran en la cuenca media y baja del río Las Ceibas, por esto y previos estudios en cultivos locales, se determinó que es posible establecer un sistema agroindustrial de Lippia alba que tenga como objetivo el 10\% del mercado de los laboratorios de productos naturales, bajo una estructura social y organizativa productiva, de transferencia de tecnología, de transformación y comercialización.

\section{Aspecto institucional}

Para el fortalecimiento y puesta en marcha de la propuesta, se identificaron que diversos actores institucionales pueden contribuir al desarrollo del sistema agroindustrial de la cadena productiva de Lippia alba en la cuenca media y baja del río Las Ceibas; tal como se muestra en la figura 1 , es importante tener en cuenta el papel que desempeñan cada uno de los diversos actores, ya que a partir de sus funciones se pueden constituir en facilitadores del proceso productivo de la planta en cuestión.

\section{Estructura social y organizativa}

Al plantear la propuesta de gestión mediante un modelo de desarrollo sostenible con las comunidades locales se pretende que los recursos se puedan enfocar 


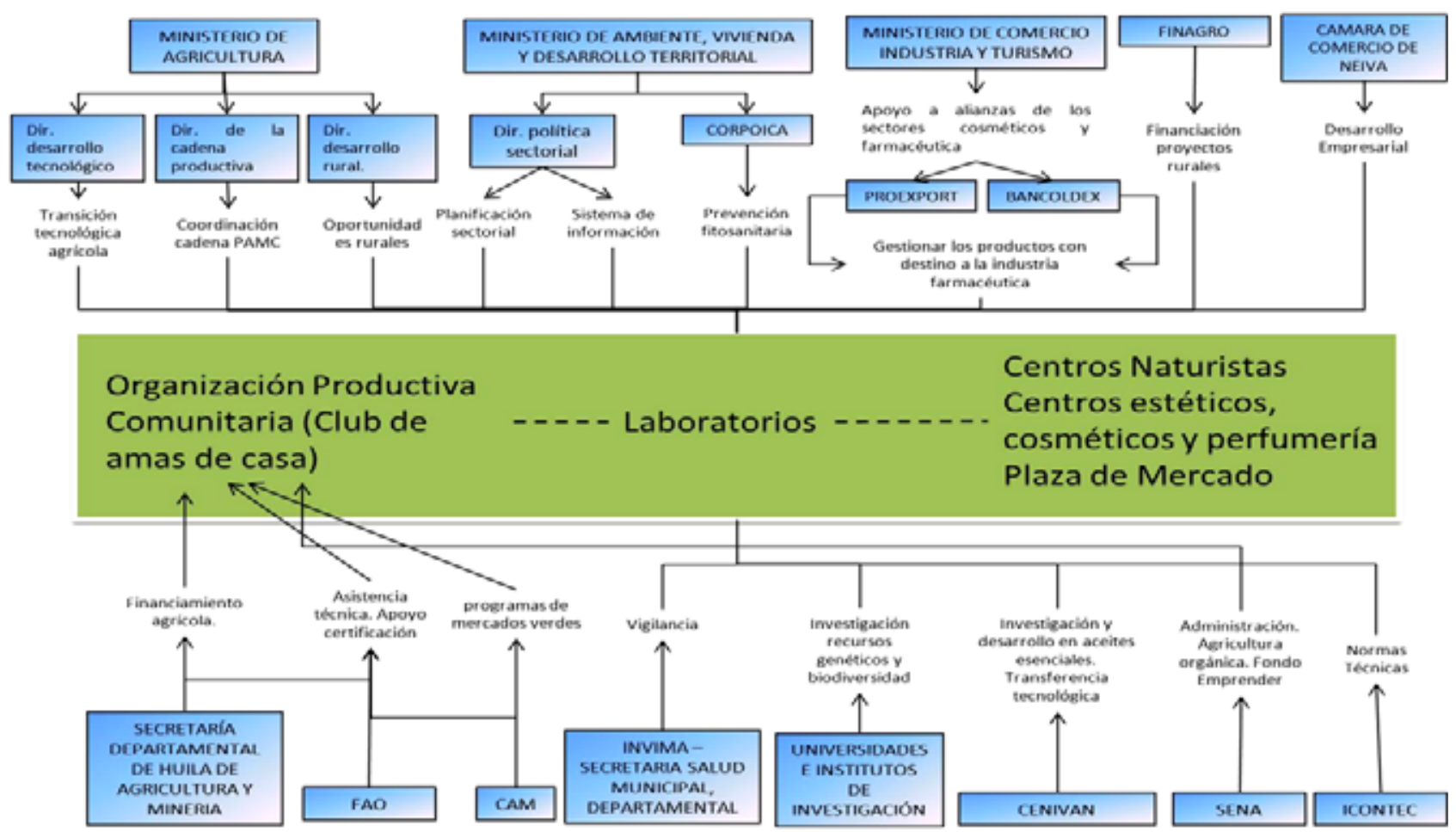

Figura 1. Mapa Institucional de la cadena productiva del Lippia alba. Adaptado de López et al. (2009)

dentro de un proyecto social y económico basado en el aprovechamiento de los recursos nativos (Baker, 2008). Además se cuenta con la oportunidad de estimular la planeación y organización local como fuerza productiva, la cual, sea la encargada de la ejecución del sistema de producción. Es decir, que es necesario involucrar de manera directa a los habitantes de la cuenca media y baja del río Las Ceibas en el aprovechamiento del recurso forestal no maderable y en este sentido, el grupo que participe en este proceso tendrá de manera paulatina la capacidad y posibilidad de administrar sosteniblemente el área protegida. También es importante recalcar que la sostenibilidad en el manejo de la cuenca incluye la generación de ingresos económicos para sus pobladores, aunque, no se pretende al menos a corto plazo, que se constituya en la fuente principal de los ingresos económicos de las familias que participen del proceso.

Siendo consecuentes, se propone que el sistema de aprovechamiento de Lippia alba sea desarrollado por un grupo comunitario que ya se encuentre constituido y que represente los núcleos familiares de la cuenca (figura 4). Es posible que este grupo sean los Clubes de Amas de Casa que existen en las veredas, puesto que uno de sus pilares está el trabajo para la generación de ingresos y la participación de las familias. Este grupo comunitario tendría como objetivo el 10\% del mercado del Lippia alba (Pronto Alivio) de los laboratorios de productos naturales, pero además habría un valor agregado para los laboratorios, que consistiría en el establecimiento de un sistema serio de comercialización.

\section{Establecimiento y escalonamiento de la producción}

El aprovechamiento agroindustrial sostenible de Lippia alba se basa en una fase productiva y en una fase de transformación primaria y comercialización. En consecuencia se plantea que la producción agrícola se instale por varias veredas pertenecientes a la cuenca baja y media del río Las Ceibas, que según el plan de ordenación y manejo de la cuenca, son terrenos pertenecientes al sistema productivo, caso contrario ocurre con la cuenca alta que es un área forestal protegida para la conservación y revegetalización. Sin embargo las zonas donde se instalaran los cultivos no deben ser alteradas significativamente y a diferencia de los otros sistemas productivos ya presentes, se utilizarán bioinsumos, biotecnología y asistencia técnica permanente proporcionando un valor agregado al producto mediante la integración entre las distintas dimensiones de sostenibilidad como la ambiental, humana, cultural, económica, político e institucional. 
En consecuencia al contar con un recurso nativo se tienen fortalezas en el ámbito de conocimiento de la planta, de sus capacidades adaptativas, y características de desarrollo dentro de un sistema productivo. Este sistema tendrá varias ventajas competitivas que le darían un valor agregado al momento de realizar la comercialización, dentro de las cuales se tienen que puede garantizar la oferta a los laboratorios interesados por tratarse de un proceso de producción y no de extracción o recolección de la planta. Así como también, que por ser un sistema agroecológico se esperaría no encontrar trazas de agroquímicos en los aceites esenciales y viabiliza un proceso de certificación de producción limpia.

Adicionalmente, el sistema productivo se establecería bajo la NTC 5400 de Buenas Prácticas Agrícolas (BPA) (ICONTEC, 2005). En este orden de ideas, no es recomendable establecer cultivos intensivos que impliquen procedimientos agrícolas que atenten contra la inocuidad del producto como son los controles químicos para las posibles plagas y enfermedades, es así que uno de los principios de la agroecología es manejar gran variedad de cultivos pequeños en las unidades productivas (Méndez, 2009; Gliessman et al., 2007; Orozco, 2006). De igual manera, los cultivos intensivos disminuyen la posibilidad de la participación comunitaria puesto que su concepción implica la concentración de las actividades agrícolas en pocos sitios, aspecto no recomendable si se quiere la mayor participación posible de los habitantes de la cuenca media y baja del río Las Ceibas, así como el ingreso a procesos de certificación en producciones limpias y posterior incorporación a mercados verdes (FAO., IAvH., 2006; RAINFOREST ALLIANCE, 2004).

De acuerdo con los argumentos anteriores, sería adecuado iniciar con el establecimiento de ocho cultivos con extensiones de $\mathbf{2 8 0 0}$ metros cuadrados cada uno, que involucren a igual cantidad de núcleos familiares como lo muestra la figura 4. Este esquema productivo permitirá la apropiación de la técnica productiva, el cumplimiento de las normas BPA y mayor participación comunitaria. A mediano plazo cuando se tengan establecidos parámetros productivos, prácticas agrológicas propias de Lippia alba, junto con canales de comercialización fortalecidos se puede ampliar la cobertura del proyecto para duplicar la producción anual. De este modo, a largo plazo se lograría vincular a 104 núcleos familiares con 27 hectáreas en producción que representarían un volumen mensual de dos toneladas deshidratadas, cantidad factible para extracción de aceites esenciales con altos estándares de calidad para exportación (Gutiérrez et al., 2004; Díaz, 2006; Ocampo y Valverde, 2000).

\section{Transformación y comercialización del producto}

El sistema de transformación primaria y comercialización inicia con el establecimiento de un centro de acopio que permita recolectar el producido de cada uno de los núcleos familiares involucrados en el sistema productivo. Este centro de acopio debe funcionar bajo los estándares de las Buenas Prácticas de Manejo (BPM) (MINISTERIO DE SALUD, 1997), esto implica recursos tecnológicos, humanos e infraestructura suficiente para garantizar el buen estado del material vegetal para su empaque y posterior entrega. También el centro de acopio debe realizar el proceso de deshidratación; de este modo, se busca que un aspecto clave como es el secado de las plantas cosechadas se realice de una manera homogénea y con las condiciones que exige el mercado de las PAMC.

De la misma manera, el presente estudio permitió establecer que existe un mercado potencial para Lippia alba con mayor rentabilidad en mercados nacionales con la venta de la planta deshidratada o fresca a laboratorios de productos naturales o farmacéuticos donde se podrían generar recursos cercanos a US $\$ 8000$ anuales, y de manera prospectiva se plantea a largo plazo la comercialización de sus aceites esenciales que posee una demanda significativa como materia prima para la industria nacional e internacional. De manera complementaria a este análisis exploratorio es necesario realizar cálculos referentes a la inversión financiera, cálculos de los costos, definición del nivel de eficiencia competitiva, costos de producción, costos administrativos, beneficios que se tendrán y evaluar el riesgo e incertidumbre.

A corto plazo (1-5 años) se plantea tener como mercado principal los laboratorios nacionales de productos naturales y farmacéuticos que compran Lippia alba deshidratado. Aunque una parte importante de la comercialización de las plantas medicinales en Colombia se realiza en las plazas del mercado, esta opción no se propone como alternativa comercial, debido a la alta informalidad que la caracteriza; esto representaría un riesgo mayor para el sistema productivo que en algún momento podría presentar iliquidez derivada de este tipo de relaciones comerciales. Amediano plazo (6-10 años) y dependiendo de la evolución del sistema productivo y de la capacidad instalada, se propone como objetivo la transformación secundaria de Lippia alba en sus aceites esenciales. Con esto se obtiene un valor 


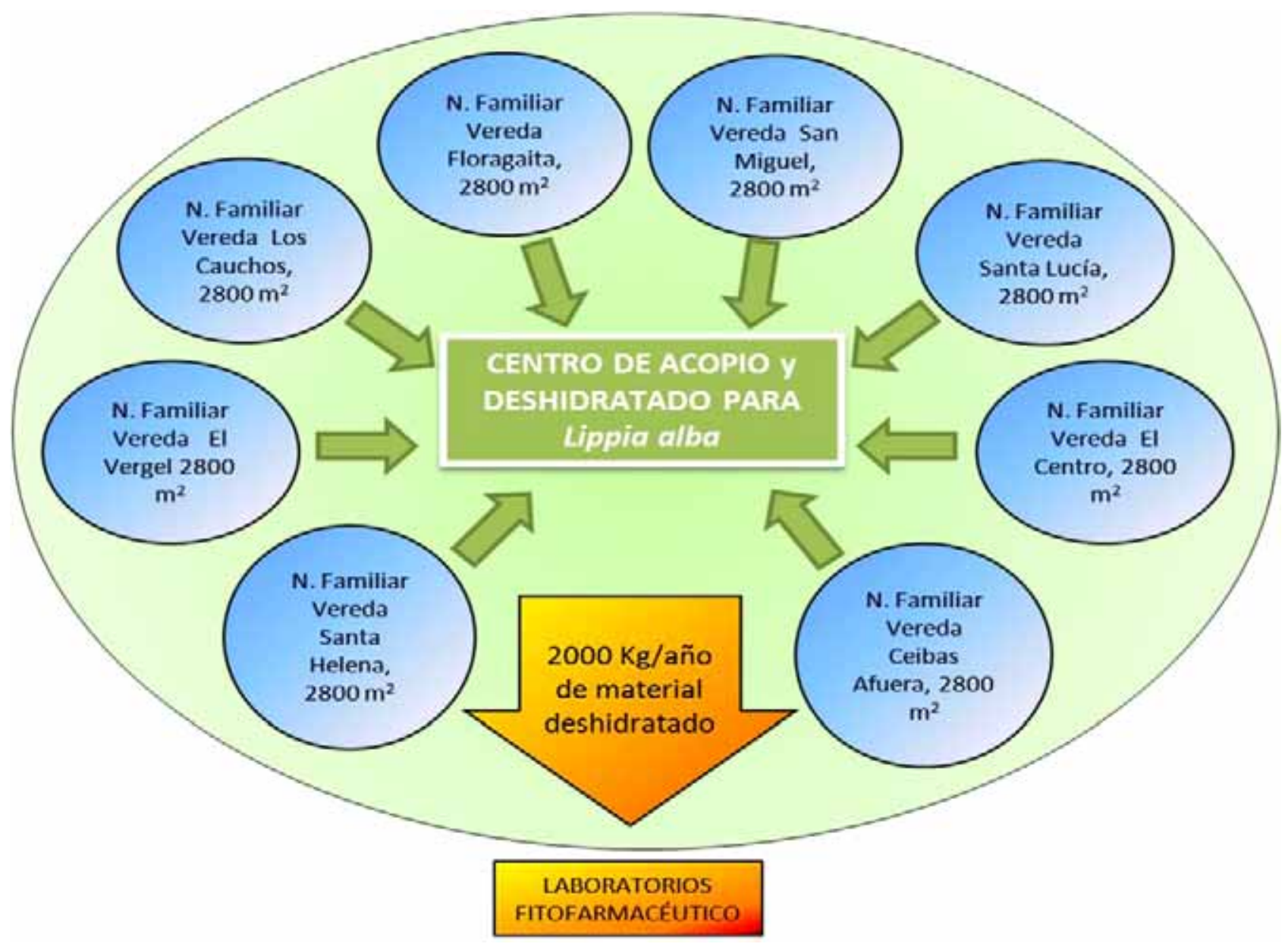

Figura 4. Esquema productivo comunitario en la cuenca media y baja del río Las Ceibas

agregado al producto y amplía las posibilidades comerciales no solo a la industria farmaceútica sino a la industria cosmética y de perfumes. A largo plazo (11 años en adelante) con la capacidad productiva, infraestructura y financiación necesaria, es posible incursionar en el mercado internacional de los aceites esenciales que contiene el Lippia alba, en este campo Brasil y Argentina se constituyen en posibles clientes, países que según reportes de la ALADI son importadores de citral, limoneno y carvona; para este fin se podría acceder a las facilidades comerciales que otorga el Mercosur a sus países miembros del cual Colombia es país asociado.

\section{Impactos}

En la búsqueda del desarrollo sustentable de la cuenca media y baja del río Las Ceibas se plantea un sistema de producción controlado, con buenas prácticas agrícolas que contribuyan a mitigar y controlar los impactos ambientales generados en su cultivo; de la misma manera, se busca fortalecer la naciente industria de las plantas medicinales y sus aceites esenciales en Colombia, con el cumplimiento de los criterios de sostenibilidad económica, ecológica y social que les permitirá a los actores de la cadena productiva de las plantas medicinales y afines satisfacer las exigencias de Buenas Prácticas de Manufactura y Calidad (PMC) en los diferentes mercados, con una distribución justa y equitativa de los costos y beneficios que se derivan de dicha producción.

\section{Conclusiones y recomendaciones}

Se identificaron que las oportunidades y limitaciones del ingreso de Lippia alba a la cadena productiva de las plantas medicinales están asociadas a varios actores de dicha cadena, al comportamiento del mercado y a las políticas existentes. Lo anterior, permitió identificar que si es viable establecer un sistema agroindustrial para dicha planta en la cuenca media y baja 
del río Las Ceibas y que su práctica además de tener como referentes normas técnicas, regulaciones sanitarias, estándares de calidad entre otras normas y legislaciones que se deben tener en cuenta principios de sostenibilidad en la producción, transformación y comercialización, puesto que son aspectos que dan valor agregado a los productos medicinales naturales.

Aunque para el año 2012 la investigación señala que las condiciones presentes en el mercado colombiano de las plantas medicinales permiten el ingreso de Lippia alba a la cadena de las PMAC, así como un potencial promisorio de sus aceites esenciales para la industria, es necesario realizar un estudio de mercado que permita establecer un plan de negocio como herramienta de planeación empresarial que identifique la dinámica de los clientes, determine el precio del producto, los costos operativos, los competidores, las alianzas, el tipo de distribución, los proveedores y describa las estrategias y acciones que se emplearán para alcanzar los objetivos propuestos.

El establecimiento de un sistema productivo de Lippia alba además de ser una oportunidad de aprovechamiento sostenible del recurso forestal no maderable presente en la cuenca del río Las Ceibas, es una posibilidad para que la agroindustria genere beneficios para los pobladores locales a partir de los servicios ambientales prestados por la diversidad biológica, mitigando los impactos negativos que las comunidades generan con los sistemas productivos tradicionales.

La alternativa propuesta requiere de un manejo especial que garantice los beneficios económicos sin llevar el sistema a la sobreexplotación del recurso biológico, por tal razón es importante el trabajo integral con la comunidad como fuerza productiva, la orientación profesional que visualice el desarrollo sostenible y las instituciones como entidades que apoyan el desarrollo rural y la conservación de los ecosistemas. Esta investigación contribuye de manera valiosa a la promoción de alternativas productivas sostenibles y a la implementación de proyectos de aprovechamiento del recurso no maderable del bosque propuestos en el Plan de Ordenación y Manejo de la Cuenca Hidrográfica del río Las Ceibas. Además la información generada realiza un aporte significativo al estudio del aprovechamiento agrosostenible de los recursos forestales no maderables en Colombia. De igual manera, se constituye en un referente para el departamento del Huila en cuanto al posible uso sostenible de un recurso biológico, ya que se expone una alternativa de gestión mediante el apoyo de disciplinas económicas aplica- das al biocomercio, con posibilidades de incursionar en los esquemas de comercialización.

Los resultados muestran que la cuenca del río Las Ceibas, cuenta con una variedad de recursos biológicos latentes para incursionar en alternativas de desarrollo sostenible. En consecuencia, las oportunidades que brinda su biodiversidad, se constituyen en razones adicionales que aportan a lo estratégico del ecosistema en cuanto al ofrecimiento de bienes y servicios ambientales se refiere; lo cual desborda su importancia más allá del servicio hídrico para abastecer el acueducto de la ciudad de Neiva.

\section{Referencias bibliográficas}

1. Baker, A. 2008. El protagonismo social de las comunidades rurales como fundamento para la cogestión adaptativa incluyente de los recursos naturales en un territorio. Centro Agronómico Tropical de Investigación y Enseñanza, CATIE: Escuela de Postgrado, Turrialba, Costa Rica. Recuperado de http:// orton.catie.ac.cr/repdoc/A2902E/A2902E.PDF

2. Barrientos, J. C., Reina, M. L., y Chacón, M. I. 2012. The economic potential of four promising aromatic species for the production of essential oils in Colombia. Revista Colombiana de Ciencias Hortícolas, 6(2), 225-237.

3. Benassini, M. 2001. Introducción a la investigación de Mercados: un enfoque para América Latina. México: Pearson Educación.

4. BIOCOMERCIO SOSTENIBLE. 2003. Estudio del mercado Colombiano de aceites esenciales. Bogotá: Instituto de Investigaciones de Recursos Biológicos Alexander von Humboldt.

5. Cardona, N. 2005. Consideraciones socioeconómicas en el diseño de proyectos sustentables de restauración ecológica. In Temas sobre restauración ecológica (Primera). México: Instituto Nacional de Ecología.

6. Ciccio, J. F., y Ocampo, R. A. 2006. Variación anual de la composición química del aceite esencial de Lippia alba (Verbenaceae) cultivada en Costa Rica. Lankesteriana, 6(3), 149-154. http://doi.org/10.15517 /lank.vi.7960

7. COLOMBIA, MINISTERIO DE SALUD. Por la cual se adoptan normas técnico-administrativas en materia 
de Medicinas Tradicionales y Terapéuticas Alternativas y se crea el Consejo Asesor para la conservación y el desarrollo de las mismas, Pub. L. No. Resolución 50781992.

8. COLOMBIA, MINISTERIO DE SALUD. Por el cual se reglamenta parcialmente la Ley 09 de 1979 y se dictan otras disposiciones, Pub. L. No. Decreto 30751997.

9. COLOMBIA, PRESIDENCIA DE LA REPUBLICA DE COLOMBIA. Por el cual se dicta el Código de los Recursos Naturales Renovables y de Protección al Medio Ambiente, Pub. L. No. Decreto 28111974.

10. COLOMBIA, PRESIDENCIA DE LA REPUBLICA DE COLOMBIA. Por el cual se reglamenta parcialmente el Régimen de Registros y Licencias, el Control de Calidad, así como el Régimen de Vigilancia Sanitaria de Medicamentos, Cosméticos, Preparaciones Farmacéuticas a base de Recursos Naturales, Productos de Aseo, Higiene y Limpieza y otros productos de uso doméstico y se dictan otras disposiciones sobre la materia, Pub. L. No. Decreto 6771995.

11. COLOMBIA. CONGRESO DE LA REPUBLICA DE COLOMBIA. Se expide el Código de Recursos Naturales y de Protección al Medio Ambiente, Pub. L. No. Ley 231973.

12. COLOMBIA. CONGRESO DE LA REPUBLICA DE COLOMBIA. Por la cual se reglamenta el uso y la industrialización de la flora medicinal, Pub. L. No. Ley 861993.

13. COLOMBIA. CONGRESO DE LA REPUBLICA DE COLOMBIA. Convenio sobre la Diversidad Biológica, Pub. L. No. Ley 1651994.

14.CONSEJO NACIONAL DE POLÍTICA ECONÓMICA Y SOCIAL, y DEPARTAMENTO NACIONAL DE PLANEACIÓN. 2010. Documento Conpes 3668: Informe Política Nacional de Competitividad y Productividad. Ley 1253 de 2008. CONSEJO NACIONAL DE POLITICA ECONÓMICA Y SOCIAL. Recuperado de https://www.dnp.gov.co/portalDNP/ politica-de-competitividad/3668.pdf

15.CONVENIO SOBRE LA DIVERSIDAD BIOLÓGICA. 2007. Examen a fondo de la aplicación del enfoque por ecosistemas (Ejecutivo No. UNEP/CBD/ SBSTTA/12/2) (p. 15). Paris: UNEP, CDB. Recuperado de http://www.cbd.int/doc/meetings/sbstta/ sbstta-12/official/sbstta-12-02-es.pdf
16.CORPORACIÓN AUTÓNOMA REGIONAL DEL ALTO MAGDALENA. 2006. Plan de Ordenación y Manejo de la Cuenca Hidrográfica del río Las Ceibas. CAM.

17.DEPARTAMENTO NACIONAL DE ESTADÍSTICAS. 2007. Documento sectorial: Cadena farmacéutica y medicamentos. Bogotá: DANE.

18.DEPARTAMENTO NACIONAL DE ESTADÍSTICAS. 2011. Índice de precios al consumidor 1996 2011. DANE. Recuperado de http://www.dane. gov.co

19.Díaz, J. 2003. Caracterización del mercado colombiano de plantas medicinales y aromáticas (p. 111). Bogotá: Instituto Alexander von Humboldt - El Ministerio de Ambiente Vivienda y Desarrollo Territorial.

20.Díaz, J. 2006. Estrategia para tres sectores de Biocomercio con estudios de mercado específicos (Vol. VII). Bogotá D. C.: Corporación Andina de Fomento, Instituto Alexander von Humboldt.

21.Durán, D., Monsalve, L., Martínez, J., y Stashenko, E. 2007. Estudio comparativo de la composición química de aceites esenciales de Lippia alba provenientes de diferentes regiones de Colombia, y efecto del tiempo de destilación sobre la composición del aceite. Scientia et Technica, 13(33), 435-438.

22. Fajardo, S. 2014. Estudio etnobotánico para la identificación del recurso forestal no maderable con mayor potencial medicinal y comercial en la cuenca media y baja del río Las Ceibas en Neiva Colombia. ENTORNOS, 27(1), 13-25.

23.Ferré, J., y Ferré, J. 1997. Los estudios de mercado: Cómo hacer un estudio de mercado de forma práctica. Madrid: Díaz de Santos, S. A.

24.Galy, S., Rengifo, E., y Hay, Y. 2000. Factores de la organización del mercado de las plantas medicinales en Iquitos, Amazonía Peruana. Folia Amazónica, 11(1), 139-158.

25.García, C., y Polanía, J. 2007. Marco conceptual para productos no maderables del bosque en manglares de Colombia. Gestión y Ambiente, 10(2), 169-178.

26.Gómez, M. 2008. Estudio de mercado de productos forestales. Turrialba, C.R.: Centro Agronómico 
Tropical de Investigación y Enseñanza (CATIE). http://orton.catie.ac.cr/repdoc/A2695E/A2695E.PDF

27. Guerrero, A. 2000. Memorias Seminario Internacional de Mercados Verdes y Ecoetiquetado una Oportunidad para la Industria Colombiana (pp. 13-16). Presentado en el Programa nacional de mercados verdes, Bogotá: Ministerio de medio ambiente, vivienda y desarrollo territorial; Universidad de los Andes. Recuperado de http://www.fidamerica.org/admin/ docdescargas/centrodoc/centrodoc_848.pdf

28. Gutiérrez, M., Duque, A., Cáceres, A., y Betancourt, Y. 2004. El mercado potencial de ocho plantas medicinales latinoamericanas. Lippia alba, Lippia graveolens, Pasiflora edulis, Petiveria alliacea, Phlebodium aureum, Quassia amara, Arrabidaea chica y Smilax domingensis (p. 32). Palmira, Colombia: OEA-AICD.

29.INSTITUTO COLOMBIANO DE NORMAS TECNICAS Y CERTIFICACIÓN. 2005. NTC 5400. Buenas prácticas agrícolas para frutas, hierbas aromáticas, culinarias y hortalizas frescas. Bogotá: ICONTEC.

30.Kinnear, T., y Taylor, J. 1998. Investigación de Mercados (5th ed.). Santa Fe de Bogotá: Mc Graw Hill.

31.LATINPHARMA. 2008. Estudio de Oferta y Demanda del Sector de Productos Naturales. PROEXPORT.

32.López, L., Mejía, D., Gómez, J., y Albarracín, C. 2009. Agenda prospectiva de investigación y desarrollo tecnológico para la cadena productiva de plantas medicinales, aromáticas, condimentarias y afines con énfasis en ingredientes naturales para la industria cosmética en Colombia. Bogotá: Ministerio de Agricultura y Desarrollo Territorial.

33. Martínez, G. 2005. Recolección y Comercialización de Plantas Medicinales en el Departamento Santa María, Provincia de Córdoba, Argentina. Journal Acta Farmacológica Bonaerense, 24(4), 575-584.

34. Martínez, R. 2006. Elementos conceptuales que apoyan las decisiones sobre el fomento de productos forestales no maderables (Ensayo) (p. 15). Bogota: University of Queesland. Recuperado de http:// www.banrepcultural.org/sites/default/files/elementos-conceptuales.pdf

35. Mesa-Arango, A. C., Montiel-Ramos, J., Zapata, B., Durán, C., Betancur-Galvis, L., y Stashenko, E.
2009. Citral and carvone chemotypes from the essential oils of Colombian Lippia alba (Mill.) N.E. Brown: composition, cytotoxicity and antifungal activity. Memorias Do Instituto Oswaldo Cruz, 104(6), 878-884. http://doi.org/10.1590/S007402762009000600010

36.Nope, C., Melo, M. M., y Rodríguez, L. F. 2008. Plan estratégico de mercadeo para el fomento de la producción de plantas medicinales y aromáticas de Asoplames (Nuevo Colón, Boyacá). Agronomía Colombiana, 26(1), 155-164.

37. Ocampo, R., y Valverde, R. 2000. Manual de cultivo y conservación de plantas medicinales (1st ed.). San José, Costa Rica.

38. Olaya, A. 2003. Los ecosistemas estratégicos del Huila según su reconocimiento legal y su área de influencia. En Ecosistemas Estratégicos del Huila: Significado ecológico y cultural (pp. 155-182). Neiva: Universidad Surcolombiana.

39. Olaya, A. 2005. Significado ecológico y sociocultural de los ecosistemas estratégicos del Huila y la región Surcolombiana, un proyecto en curso. En La USCO piensa la región: aportes desde la investigación. Neiva: Universidad Surcolombiana.

40. Olaya, A., y Sánchez, M. (Eds.). 2003. Ecosistemas estratégicos del Huila: Significado ecológico y sociocultural (1st ed.). Neiva: Universidad Surcolombiana.

41.Palacios, E. 2014. Economía y Plantas Medicinales. Pensamiento Crítico, 3(0), 011-023.

42.Patiño, A. 2004. Jerarquización y descripción de los ecosistemas estratégicos de la subregión norte del Huila según criterios ecológicos y socioculturales. Surcolombiana, Neiva.

43.PROEXPORT. 2003. Estudio de oferta y demanda del sector de productos naturales. Bogotá: PROEXPORT.

44.Puelles, M. 2008. Estudio de la viabilidad comercial de plantas medicinales en zonas rurales altas del Valle del Mantaro (Perú) (Resumen de resultados y conclusiones) (pp. 2-10). Madrid: Universidad de Complutense. Recuperado de http://www.reduniver sitaria.es/investigacion/PlantasMedicinales-res.pdf

45. REVISTA DINERO. 2005. Un mercado natural. Revista Dinero, (223), 7-8. 
46. SECRETARÍADEL CONVENIO SOBRE LADIVERSIDAD BIOLÓGICA. 2004. Enfoque por ecosistemas. Montreal: PNUMA, CDB. Recuperado de https://www.cbd.int/doc/publications/ea-text-es.pdf

47.UNITED NATIONS CONFERENCE ON TRADE AND DEVELOPMENT. 2005. Market Brief in the European Union for selected natural ingredients derived from native species: Lippia alba. Switzerland: UNCTAD / BioTrade Facilitation Programme. Recuperado de http://www.biotrade.org/Resources Publications/biotradebrief-lippiaalba. pdf
48. Van Hoof, B. 2000. Memorias Seminario Internacional de Mercados Verdes y Ecoetiquetado una Oportunidad para la Industria Colombiana (pp. 29-42). Presentado en Las oportunidades que brindan los mercados verdes a la industria colombiana, Bogotá: Ministerio de medio ambiente, vivienda y desarrollo territorial; Universidad de los Andes. Recuperado de http:// www.fidamerica.org/admin/docdescargas/centro doc/centrodoc_848.pdf

49. Vega, M. 2001. Etobotánica de la Amazonía Peruana. Quito: Abya-Yala. Recuperado de http://repository. unm.edu/bitstream/handle/1928/11667/Etnobot\% C3\%A1nica\%20de\%20la\%20amazon\% C3\%ADa\% 20peruana.pdf?sequence $=1$ 
